www.jmscr.igmpublication.org

Impact Factor 5.84

Index Copernicus Value: 83.27

ISSN (e)-2347-176x ISSN (p) 2455-0450

crossref DOI: https://dx.doi.org/10.18535/jmscr/v5i7.141

Journal Of Medical Science And Clinical Research

\title{
Efficiency of Single Dose Antibiotic Prophylaxis in Inguinal Hernioplasty
}

\section{Authors}

\section{Pavan BK ${ }^{1}$, Bhaskaran A $^{2}$, Asadulla Baig ${ }^{3}$, Akarsh YG $^{4}$, Karthik Hareen TVK}

${ }^{1}$ Asst Professor, Dept of General Surgery, Sri Devraj Urs Medical College, Kolar

${ }^{2}$ Professor, Dept of General Surgery, Sri Devraj Urs Medical College, Kolar

${ }^{3}$ Asst Professor, Dept of General Surgery, Sri Devraj Urs Medical College, Kolar

${ }^{4}$ Senior resident, Dept of General Surgery, Sri Devraj Urs Medical College, Kolar

${ }^{5}$ Post-graduate, Dept of General Surgery, Sri Devraj Urs Medical College, Kolar

\section{ABSTRACT}

Introduction and objectives: Surgical site infection (SSI) is the most frequent complication in inguinal hernioplasty.SSI is related with an increase in length of stay and costs and a decrease in quality of life. Use of antibiotics in Lichtenstein's hernia repair is still debatable in a rural set up. Another subject in rural setup that must be addressed in antibiotic prophylaxis is cost-effectiveness. In these cases, the costs of antibiotic administration must be carefully evaluated against the potentials benefits.

Materials and Methods: The study was conducted out between December 2014 to October 2016 on 60 patients. The patients were assigned alternately into 2 groups. Cases in Group A received single dose of pre-operative antibiotic 30 minutes before surgery and cases in group B received multiple doses perioperatively. The patients were then monitored post operatively from the second day onwards for SSI. The data was collected and statistics done using the Chi square/Fischer exact test to look for significant difference in the rate off SSIs in both the groups and also to find out if it was cost effective.

Results and Inferences: Incidence of SSI in group A was 3.33\% (1 in 30). Incidence of SSI in group B was $6.6 \%$ ( 2 in 30).Incidence of SSI though higher in group B than group A was not statistically significant when Fischer exact test was applied. The cost of antibiotic per patient in group A was Rs.32 while that in group B was between a minimum of Rs.192 in patients without SSI to a maximum of Rs.340 with SSI. The difference in cost of antibiotic in both groups was statistically significant.

Conclusion: Single dose antibiotic prophylaxis was therapeutically efficient as well as cost effective in comparison with multiple doses of perioperative antibiotics usage for the prevention of surgical site infection in uncomplicated elective cases of Lichtenstein's hernioplasty.

Keywords: inguinal hernia; lichtenstein's repair; SSI; antibiotic; prophylaxis.

\section{INTRODUCTION}

Surgical site infection (SSI) is the most frequent complication in inguinal hernioplasty ${ }^{1}$. The risk factors for SSI have been identified as sex (greater in women), age (older than 70 years), co morbidity, operative time, and routine use of drainage and prostheses ${ }^{2-5}$. SSI is related with an increase in length of stay and costs and a decrease in quality of life ${ }^{6}$. However, the introduction of free tension hernioplasty has made the use of antibiotic prophylaxis more critical because of the infection risk when prosthetic materials are used.The presence of plastic biomaterial increases the incidence of complications relating to the 
mesh itself, in addition to other recognized complications of the hernia repair ${ }^{7}$.

To prevent mesh infection, antibiotic prophylaxis is often indicated and recommended. The incidence of mesh infection of $1.9 \%$ to $7.5 \%$ has been reported ${ }^{7}$. Available evidence related to the effectiveness of antibiotic prophylaxis for inguinal hernioplasty is found in a meta-analysis, including few RCTs. SSI rate was $1.2 \%$ and $3.3 \%$, in the prophylaxis and placebo group respectively. These results concluded there were no statistical differences between groups ${ }^{8}$. A previous metaanalysis by Sanchez-Manuel and Seco-Gil for the Cochrane Collaboration, including 8-high quality RCTs, reported no statistical difference in SSI rates between antibiotic and no antibiotic groups. However, a subgroup analysis suggested that, in mesh hernia repair, a protective effect could exist, undetectable because of the small sample size ${ }^{9}$.

Use of antibiotics in Lichtenstein's hernia repair is still debatable in a rural set up. Some surgeons use a single dose of pre-operative antibiotic, while the majority use multiple doses of post-operative antibiotics claiming that the latter is superior to the former in prevention of SSI in Lichtenstein's repair. Another subject in rural setup that must be addressed in antibiotic prophylaxis is costeffectiveness. In these cases, the costs of antibiotic administration must be carefully evaluated against the potentials benefits ${ }^{10-14}$. Only studies particularly designed to answer this question could solve it. This study is intended for the above reasons.

\section{MATERIALS AND METHODS DESIGN}

A prospective comparative study

\section{COLLECTION OF DATA AND SOURCES}

1. The study was conducted in R.L.Jalappa Hospital and Research centre and SNR Hospital, Kolar on 60 patients from December 2014 to October 2016.

2. The population for the study were from surgical units in R.L.Jalappa Hospital, Tamaka, Kolar and SNR hospital,Kolar.
CRITERIA FOR SELECTION OF STUDY GROUP

\section{A. Inclusion criteria}

- Adult patients with primary inguinal hernia.

\section{B. Exclusion criteria}

- Paediatric age group

- Patients with recurrent and complicated hernias

- Immunocompromised patients

- Patients with skin and soft tissue infections

- Patients who have received antibiotics within past 48 hours

- Patients with cardiac valvular disease and prosthetic valves

- Patients with uncontrolled Diabetes Mellitus and Hypertension

\section{GROUP SELECTION}

Alternate cases were assigned into two groups A and $\mathrm{B}$.

1. Group A received 1 gram of Cefazolin intravenously 30 minutes before the surgical incision.

2. Group B received 1 gram of Cefazolin intravenously peri-operatively and twice daily post operatively for 3 days

\section{FOLLOW UP}

The surgical site was inspected daily from second post-operative day onwards based on the following criteria for SSI.

\section{SSI SURVEILLANCE CRITERIA}

1. Presence of erythema, extending at least $2 \mathrm{cms}$ beyond the wound edges and purulent discharge.

2. Wound dehiscence.

When there was no SSI, sutures were removed on the $7^{\text {th }}$ post-operative day and the patient was discharged. In patients who had SSI, culture and sensitivity tests were done and appropriate antibiotics were given.

\section{DATA COLLECTION AND ANALYSIS}

The following data were collected and analysed.

1. Patients demographic profile 
2. Clinical type of hernia (direct / indirect)

3. Biochemical parameters

4. Anaesthesia variables such as

- ASA grade

- Type of anaesthesia

- Duration of anaesthesia

\section{STATISTICAL ANALYSIS}

1. Analysis of the data was done with primary objective to find out if single dose of pre-operative antibiotic is cost effectively more beneficial than those receiving multiple doses of peri-operative antibiotic in preventing surgical site infections after Lichtenstein's repair.

2. Association between a group and a SSI will be assessed using Chi square test/ Fischer exact test.

\section{RESULTS}

60 patients were recruited to the study from December 2014 to October 2016. Patients were assigned into two groups $A(n=30)$ and $B(n=30)$, to receive single dose or multiple dose antibiotics respectively. The baseline characteristics were similar in both groups. There was no statistically significant age specific infection risk. Type of anaesthesia, and ASA grade were similar in both groups. Average duration of procedure was almost 50 minutes to 90 minutes in all the patients. SSI was identified on the $3^{\text {rd }}$ to $4^{\text {th }}$ post-operative day in both groups. Bacteria isolated were Klebsiella, staphylococcus and E.coli. Incidence of SSI in group A was $3.33 \%$ (1 in 30) Incidence of SSI in group B was $6.6 \%$ (2 in 30).Incidence of SSI though higher in group B than group A was not statistically significant when Fischer exact test was applied. Incidence of SSI was higher in both the groups with higher ASA Grade and prolonged duration of surgery. The cost of antibiotic per patient in group A was Rs.32 while that in group B was between a minimum of Rs.192 in patients without SSI to a maximum of Rs.340 with SSI. The difference in cost of antibiotic in both groups was statistically significant.

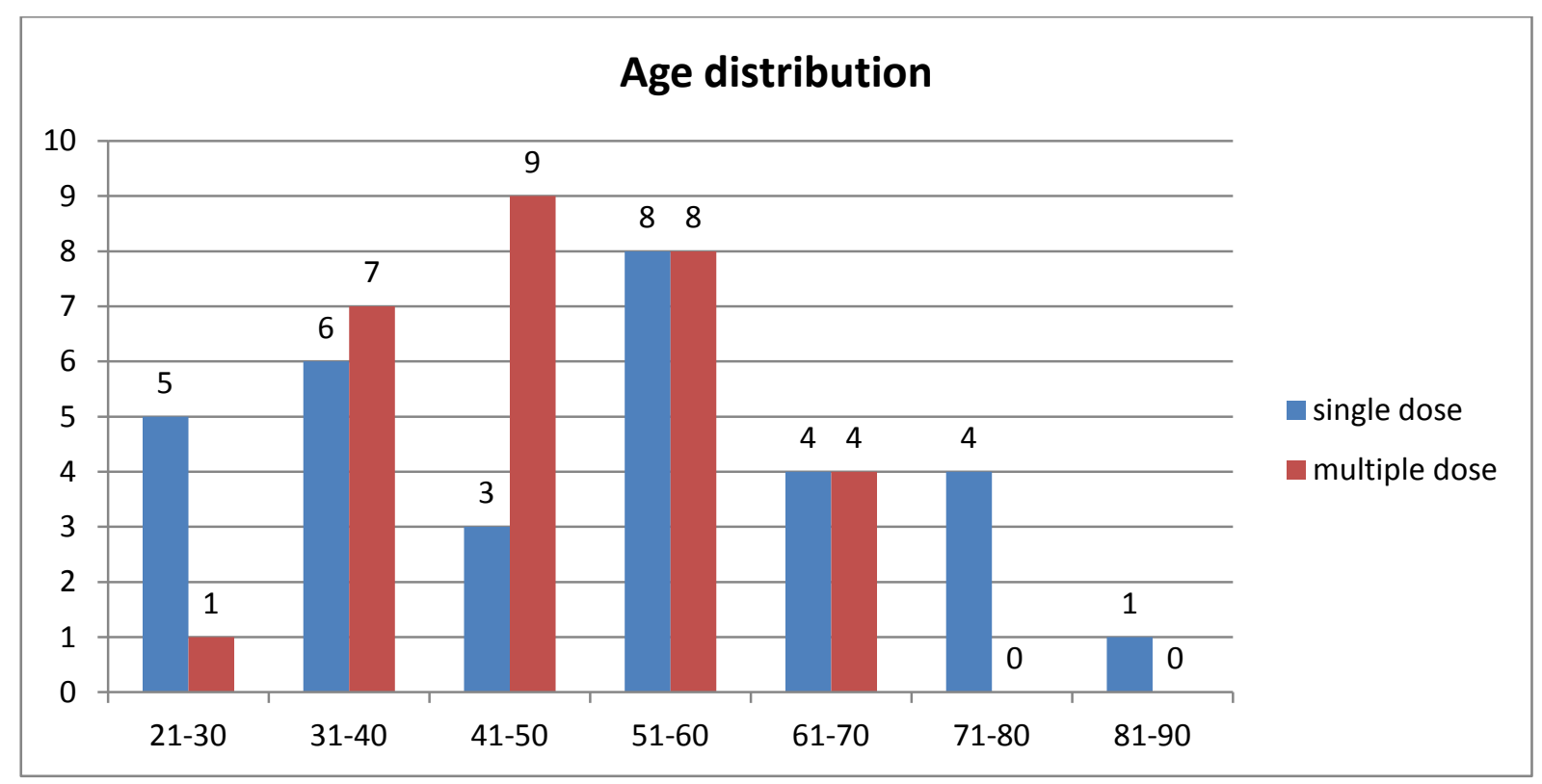



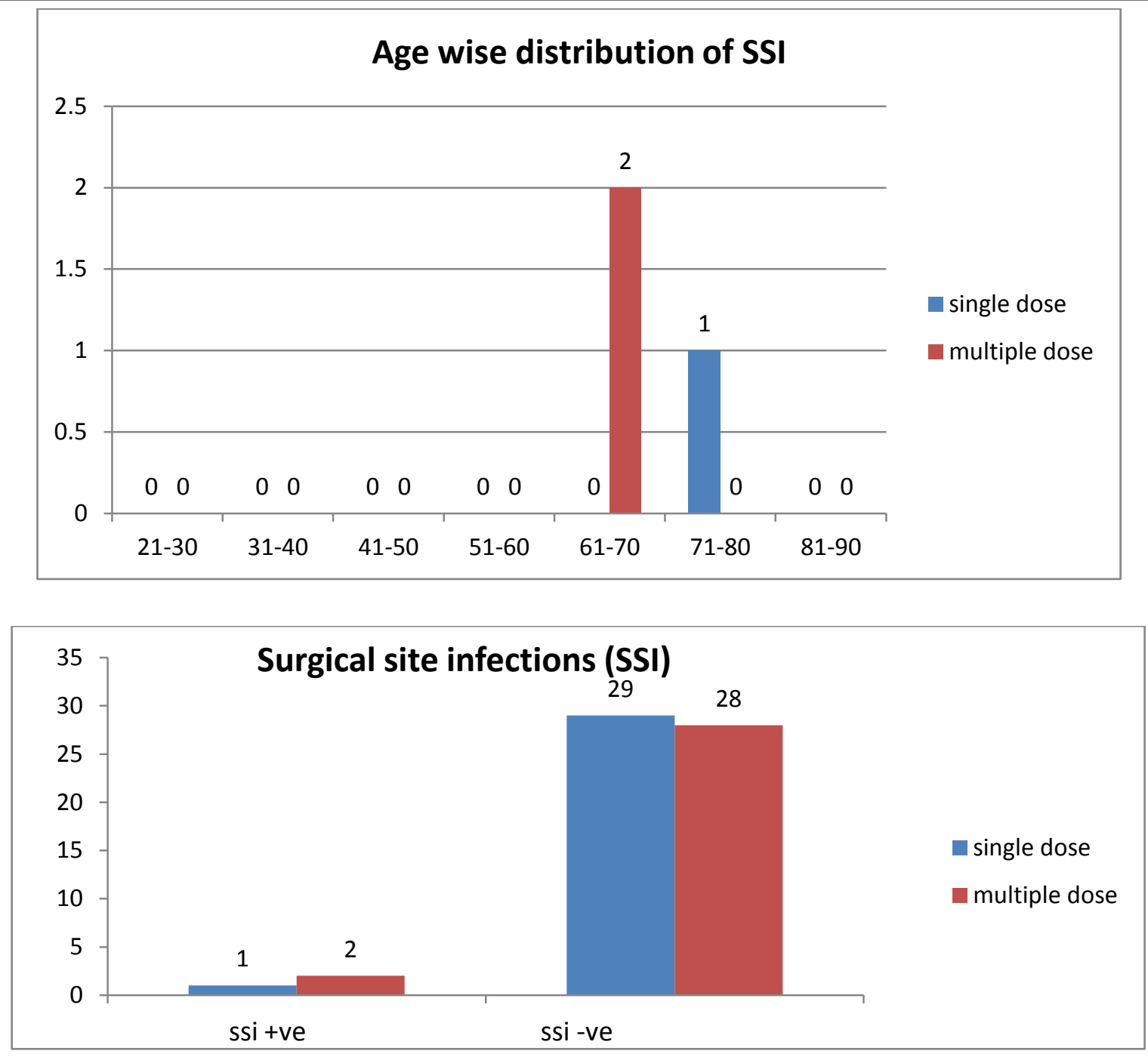

\begin{tabular}{|l|c|c|c|c|}
\hline S1 no. & Group & Age & Detected on pod & Organism \\
\hline 1 & Single dose & 75 & 3 & Staph aureus \\
\hline 2 & Multiple dose & 70 & 4 & Klebsiella \\
\hline 3 & Multiple dose & 65 & 3 & E .coli \\
\hline
\end{tabular}

\begin{tabular}{|l|c|c|c|c|c|c|}
\hline Cost (independent $\mathrm{t}$ test) & & & & & \\
\hline Group & $\mathrm{N}$ & Mean & St. Dev & degree of freedom & $\mathrm{t}$ value & $\mathrm{p}$ value \\
\hline single dose & 31 & 36.1 & 23 & 48 & -21.64 & $<0.001$ \\
\hline multiple dose & 29 & 201.2 & 34.5 & & & \\
\hline
\end{tabular}

\section{DISCUSSION}

Inguinal hernia is the commonest problem amongst all external hernias and inguinal hernia repair is most frequent procedure in general surgery accounting for $10-15 \%$ of all operations. The age incidence is distributed in all decades of life. Incidence of inguinal hernia is race related. It is at least three times more common in black Africans than in the white population.
About $80-90 \%$ of repairs are done in males. The most frequent type is right sided indirect inguinal hernia. Direct inguinal hernias are rare in females. Due to its common nature and increased incidence of recurrence and wound infection, a wide variety of surgical procedures and different materials were being used from time to time for hernia repair. 
All these procedures and materials have equivocal results and are beyond the level of satisfaction for different surgeons. All these modifications and surgical techniques have showed a common disadvantage i.e. suture line tension, which leads to increased incidence of recurrence and other complications. Post-operative wound infection remains a common complication after hernia repair. With the use of modern mesh prosthesis, it is now possible to repair all hernias without distortion of the normal anatomy and with no suture line tension.

Modern mesh is strong monofilament, inert, and readily available. It is unable to harbour infection, is very thin and porous. Its interstices become completely infiltrated with fibroblasts and remain strong permanently .It is not subjected to deterioration or rejection or it cannot be felt by patients or surgeons postoperatively. Many factors including antimicrobial prophylaxis affect surgical site infection. For example, beginning antibiotic prophylaxis during the immediate preoperative period reduces the risk of wound infection fourfold. Maintaining therapeutic antibiotic levels in the serum and tissues throughout the operation until, at most, a few hours after incision closure reduces this risk. Both in the US and Europe,more than 1 million inguinal hernia repair are performed anually, of which Lichenstein's open meshplasty is the most popular. A trial conducted in 2004 showed that infection rate was low (1.7\%)after Lichtenstein meshplasty ${ }^{15}$.Another study conducted by Najam-ul-Haq R et al. in 2004 showed that the overall incidence of postoperative complications was $11 \%$, all complications were treated conservatively and removal of mesh was not required postoperatively $^{16 .}$

In another study conducted in 2005 byDelikoukos $\mathrm{S}$, to report late mesh infection occurring years after open hernioplasty, it was proved that late graft infection does not correlate to neither the administration or not of antibiotic prophylaxis nor to the presence or not of previous superficial wound infection ${ }^{17}$. The debate still continues as to the clinical use of antibiotic prophylaxis and stratification of the risk factors so as to help differentiate between the high and low risk patients. A meta-analysis conducted by Sanabria Aet al. in 2007 showed that prophylactic antibiotic in hernioplasty reduced the rate of SSI by $50 \% .^{18}$

A randomized double blind placebo controlled study conducted by Thakur et al in 2008 showed that $10.3 \%$ of the patients in the antibiotic group and $15.3 \%$ of patients in the placebo group had wound infections, proving that there was no stasitically significant difference observed between the two groups ${ }^{19}$

In this study, patients in Group A received a single dose of Inj. Cefazolin $1 \mathrm{~g}$ at induction time and patients in Group B received perioperative doses of Inj. Cefazolin $1 \mathrm{~g}$, twice daily for 3 days Indiscriminate use of antibiotics leads to proliferation of resistant organisms and was probably responsible for high rate of surgical site infection of $6 \%$ in Group B when compared with group A 3.3\%. Pathogens encountered in these patients were E.coli, Staphylococcus aureus, Klebsiella.

\section{CONCLUSION}

Single dose antibiotic prophylaxis was therapeutically efficient as well as cost effective in comparison with multiple doses of postoperative antibiotics usage for the prevention of surgical site infection in uncomplicated elective cases of Lichtenstein's hernioplasty. The study shows that the cost of management of hernia patients with respect to use of antibiotics can be reduced in a rural set up by use of single dose antibiotic, thereby reducing financial burden on the patient.

\section{REFERENCES}

1. Bendavid R. Complications of groin hernia surgery. Surg Clin North Am. 1998;78:1089-1103

2. Abo RE. Perioperative antibiotic prophylaxis in abdominal surgery for hernia repair: retrospective study of 1,524 
consecutive patients. J Chemother. 1998;10: 248-253.

3. Taylor EW, Duffy K, Lee K, et al. Surgical site infection after groin hernia repair. Br J Surg. 2004;91:105-111.

4. Deysine M. Pathophysiology, prevention, and management of prosthetic infections in hernia surgery.SurgClin North Am1998;78:1105-1115.

5. Amid PK. Classification of biomaterials and their related complications in abdominal wall hernia surgery. Hernia. $1997 ; 1: 15-21$.

6. Barie PS. Modern surgical antibiotic prophylaxis and therapy-less is more. Surg Infect (Larchmt). 2000; 1:23-29.

7. Patino JF, Garcia-Herreros LG, Zundel N. Inguinal hernia repair: the Nyhus posterior preperitoneal operation. SurgClin North Am. 1998;78:1063-1074.

8. Weed HG. Antimicrobial prophylaxis in the surgical patient. Med Clin North Am. 2003;87:59-75]

9. Sanchez-Manuel FJ, Seco-Gil JL. Antibiotic prophylaxis for hernia repair. Cochrane Database Syst Rev. 2004;CD003769

10. Lewis RT, Weigand FM, Mamazza J, et al. Should antibiotic prophylaxis be used routinely in clean surgical procedures: a tentative yes. Surgery. 1995;118:742-746.

11. Platt R. Antibiotic prophylaxis in clean surgery: does it work? Should it be used if it does? New Horiz. 1998;6 (suppl):53-57.

12. D'Amico DF, Parimbelli P, Ruffolo C. Antibiotic prophylaxis in clean surgery: breast surgery and hernia repair. J Chemother. 2001 ; 13:108-111.

13. Gupta R, Sinnett D, Carpenter R, et al. Antibiotic prophylaxis for post-operative wound infection in clean elective breast surgery. Eur J SurgOncol. 2000;26:363366.
14. Cheek CM, Williams MH, Farndon JR. Trusses in the management of hernia today. Br J Surg. 1995;82:1611-1613.

15. Aufenacker JT,Geldere D,Mesdag T.The role of antibiotic prophylaxis in prevention of wound infection after Lichtenstein open mesh repair of primary inguinal hernia .Ann Surg 2004;240:955-961.

16. Najam-ul-Haq R,Chaudhry IA,Khan BA,Afzal M.Groin prophylaxis following hernioplasty without antibiotic prophylaxis-a review of 100 cases.Pak J Med Sci 2006;4:416-419.

17. Delikoukos S,Tzovaras G,Liakou P,Mantzos F,Hatzitheofilou C.Late onset deep mesh infection after inguinal hernia repair.Hernia 2007;11:15-17.

18. Sanabria A,Doniguez C L,Valdivieso E,Gomez G. Prophylactic antibiotics for mesh inguinal hernioplasty.AnnSurg 2007; 245: 392-396.

19. Thakur L,Uphadhyay S,Peters J N.Prophylactic antibiotic usage in patients undergoing inguinal mesh hernioplasty-A clinical study.IJS;72:240-242. 